1 Universidade Federal de Pelotas (UFPEL), Faculdade de Enfermagem - Pelotas (RS), Brasil.

polibrina@hotmail.com

2 Universidade Federal de Pelotas (UFPEL), Faculdade de Enfermagem - Pelotas (RS), Brasil.

kantorski@uol.com.br

3 Universidade Federal de Pelotas (UFPEL), Faculdade de Enfermagem - Pelotas (RS), Brasil.

valeriacoimbra@hotmail.

com

4 Universidade Federal de Pelotas (UFPEL), Faculdade de Enfermagem - Pelotas (RS), Brasil.

mandagara@hotmail.com

5 Universidade Federal de Pelotas (UFPEL), Faculdade de Enfermagem - Pelotas (RS), Brasil.

kaa_langmantel@hotmail. com

\section{Indicadores qualitativos de satisfação em saúde mental}

\author{
Qualitative indicators of satisfaction in mental health
}

\author{
Poliana Farias Alves', Luciane Prado Kantorski², Valéria Cristina Christello Coimbra ${ }^{\mathbf{3}}$, Michele \\ Mandagará de Oliveira ${ }^{4}$, Karine Langmantel Silveira ${ }^{5}$
}

RESUMO Este artigo objetivou construir indicadores qualitativos de resultado relacionados com a satisfação na perspectiva dos usuários e familiares. Estudo de caso avaliativo, participativo, hermenêutico-dialético, realizado em um Centro de Atenção Psicossocial no Rio Grande do Sul, com usuários e familiares em grupos focais para discussão, negociação e validação de indicadores qualitativos em 2014. Identificaram-se dois indicadores qualitativos de satisfação, sendo estes: a não internação psiquiátrica ou menor necessidade desta; sentimento ou sensação de bem-estar. Conclui-se que usuários e familiares apresentam-se satisfeitos com a práxis do Centro de Atenção Psicossocial estudado.

PALAVRAS-CHAVE Saúde mental. Serviços comunitários de saúde mental. Avaliação. Indicadores. Avaliação de serviços de saúde.

ABSTRACT This article aimed to build outcome qualitative indicators related to satisfaction from the perspective of users and relatives. Evaluative case study, participatory, hermeneutic-dialectical, performed in a Psychosocial Care Center, in Rio Grande do Sul, with users and relatives in focal groups for discussion, negotiation and validation of qualitative indicators in 2014. We identified two qualitative indicators of satisfaction: non-psychiatric hospitalization or less need of it; the feeling or sense of well being. It was concluded that users and relatives presented satisfaction with the praxis from the Psychosocial Care Center studied.

KEYWORDS Mental health. Community mental health services. Evaluation. Indicators. Health service evaluation. 


\section{Introdução}

Ao longo dos anos, no campo da saúde mental, o Movimento da Reforma Psiquiátrica vem ganhando força, apoio e mais aliados à causa, trazendo novos conceitos para o Brasil. Consequentemente, todo esse movimento de reforma vem sendo palco de discussões sobre a saúde mental, de conscientização, elaboração de novas alternativas aos problemas relacionados à saúde mental brasileira e à incorporação de novos conceitos como os da desinstitucionalização e da atenção psicossocial.

O modo psicossocial, sinteticamente, pode ser conceituado como um modo de cuidado em saúde mental baseado na superação das práticas historicamente instaladas. Ele apresenta propostas fundamentais para suas ações, como: o deslocamento do local de execução das práticas em saúde mental do interior da instituição para o território com foco na integralidade do cuidado, a superação do paradigma de adequação do usuário ao meio a partir dos tratamentos, o desenvolvimento de ações que produzam subjetividade singularizada e a horizontalização das relações interprofissionais e com os usuários. Essas propostas fundamentais da atenção psicossocial constituem base para superar o modo de relação sujeito-objeto, característico do modelo médico e das ciências positivas, em que se baseou o cuidado em saúde mental por muitos séculos (COSTA-ROSA; LUZIO; YASSUI, 2001).

A criação, regulamentação e expansão de serviços de cuidado em saúde mental, também conhecidos como Centros de Atenção Psicossocial (Caps), ocorreram a partir de 2001 e têm sua praxis baseada nos conceitos da atenção psicossocial (BRASIL, 2001; BRASIL, 2002). Para que se possa analisar a sua eficácia, é veemente a necessidade de avaliações desses serviços, principalmente no que concerne à satisfação dos usuários com a qualidade das ações prestadas.

Atualmente, uma das formas mais utilizadas para avaliar a qualidade de um serviço é a partir do conhecimento da satisfação da população atendida por ele. De acordo com Bandeira, Pitta e Mercier (2000), a satisfação dos usuários com serviços de saúde vem sendo, na sua grande maioria, avaliada quantitativamente por meio de escalas validadas no Brasil, a exemplo das Escalas Brasileiras de Avaliação da Satisfação (Satis-BR) validadas por essas mesmas autoras, para a construção de indicadores na área.

Os indicadores são uma espécie de sinalizadores da realidade, podendo estes ser quantitativos ou qualitativos. Eles servem para afirmar ou não se os objetivos e os resultados de uma política ou serviços estão sendo bem conduzidos ou alcançados (MINAYO, 2008).

A construção de indicadores quantitativos se caracteriza como proveniente da lógica quantitativa, principalmente por meio de escalas. Contudo, essa forma de construção de indicadores, em geral, reduz a questão da singularidade e da individualidade às proposições estabelecidas na perspectiva do pesquisador, resultando em indicadores que permanecem externos aos sujeitos investigados. Por outro lado, a construção de indicadores qualitativos por meio de estratégias qualitativas possui a capacidade de expressão da voz, sentimentos, pensamentos e práticas desenvolvidas pelos atores que estão envolvidos no processo avaliativo (MINAYO, 2008).

Os indicadores de resultados fornecem informações sobre a eficácia dos serviços de saúde, porém, apenas poucos estudos têm construído e utilizado indicadores de resultado para comparar e melhorar a qualidade entre esses serviços (PERLMAN ET AL., 2013).

Neste estudo, o argumento central se apoia na natureza hermenêutica para a construção dos indicadores qualitativos, na emergência destes a partir da realidade empírica e concreta e na importância de colocar os usuários e familiares no centro de processos avaliativos. Parte-se do entendimento de que os resultados das ações desenvolvidas pelos serviços de saúde mental na vida dos usuários e familiares têm que ser observados a partir da perspectiva deles. 
Guba e Lincoln (2011) são precursores da utilização do referencial teórico hermenêutico-dialético como base para o desenvolvimento de avaliações. É hermenêutico porque tem caráter interpretativo e dialético porque implica comparação e contraste de diferentes pontos de vista. Assim, a justificativa para a utilização desse referencial é que ele leva a uma exploração mútua de todas as partes interessadas no processo avaliativo para alcançar um consenso entre os interessados, dando voz aos usuários e familiares.

$\mathrm{O}$ intuito de construção de indicadores qualitativos de resultado a partir de uma avaliação de serviço vem da preocupação em apreender as implicações do Caps na vida dos usuários e dos familiares, a forma como os atores interagem entre si, os sentidos que são construídos por estes em relação à prática exercida, bem como a satisfação com os resultados.

A temática das mudanças percebidas pelos pacientes e pelos seus familiares se mostra de grande importância para a avaliação dos efeitos do tratamento recebido nesses serviços, uma vez que integra diferentes percepções e expectativas na avaliação da eficácia do tratamento (COSTA ET AL., 2011). Neste estudo, destaca-se a importância de analisar os resultados oriundos do cuidado dispensado nos serviços de saúde mental, porém a partir de uma metodologia qualitativa, em que se pretende apreender aspectos mais subjetivos relacionados à satisfação. Não se trata aqui de relatar se está satisfeito ou não com determinado aspecto, mas, mais que isso, é de escutar o porquê de estar satisfeito com determinado aspecto, o que dá ao processo avaliativo um aprofundamento sobre o que se está analisando.

Este estudo teve por objetivo construir indicadores qualitativos de resultado relacionados à satisfação na perspectiva dos usuários e de familiares a partir da inserção destes em um Caps partindo do pressuposto de que a satisfação se caracteriza como uma variante que poderia ou deveria ser utilizada para avaliar a eficácia de serviços de saúde.

\section{Metodologia}

Este estudo se caracteriza como um estudo de caso avaliativo, de abordagem construtivista, a partir de um referencial teórico hermenêutico-dialético com o intuito de construir indicadores qualitativos acerca da temática 'satisfação na atenção psicossocial'. Ele é oriundo de uma dissertação de mestrado e foi realizado em um Caps no município de Alegrete, no estado do Rio Grande do Sul.

Para a realização deste estudo, partiu-se de duas pesquisas anteriormente realizadas na região Sul do Brasil, a primeira, Avaliação dos Caps da Região Sul do Brasil (Capsul I), no ano de 2006, e a segunda, Capsul II (Segunda Avaliação dos Caps da Região Sul do Brasil), no ano de 2011 (KANTORSKI, 2007; KANTORSKI, 2011). Tais pesquisas tiveram como objetivo avaliar os serviços de saúde mental nos estados do Paraná, Santa Catarina e Rio Grande do Sul totalizando 308 serviços avaliados quantitativamente e 3 serviços avaliados qualitativamente baseado na avaliação de quarta geração.

Guba e Lincoln (2011) apontam a avaliação de quarta geração como alternativa às tradicionais formas utilizadas para avaliar serviços, entendendo que o foco organizacional do serviço deve partir das reivindicações, preocupações, necessidades e questões dos grupos de interesse.

Para o desenvolvimento deste estudo de construção dos indicadores qualitativos, a coleta de dados foi dividida em duas etapas descritas a seguir.

A primeira etapa ocorreu de fevereiro a julho de 2014 e consistiu em retomar o banco de dados qualitativos do processo avaliativo das pesquisas Capsul I e Capsul II do município de Alegrete (RS). Tal banco é composto por: diários de campo, entrevistas de usuários e entrevista de familiares, totalizando 46 documentos. Iniciou-se por uma análise sistematizada desses dados, que teve por objetivo identificar nas falas de usuários e familiares relatos referentes às mudanças 
ocorridas em suas vidas a partir da inserção destes no serviço de saúde mental estudado, relatos estes que contribuíssem para a construção de indicadores de avaliação da atenção psicossocial em Caps. Essas falas foram organizadas em uma matriz, constituindo unidades de informação. A partir da análise e interpretação destas, foram criados 20 possíveis indicadores qualitativos de resultado com base no marco conceitual utilizado, o da atenção psicossocial. Essas unidades de informação e seus respectivos indicadores foram arranjados na matriz de acordo com a homogeneidade dos seus temas constituindo categorias provisórias, sendo estas: Autonomia, Reinserção Social, Preconceito e Satisfação.

A segunda etapa da coleta ocorreu de 18 de agosto a 2 de setembro de 2014. Ela consistiu em retornar ao serviço estudado para realização da técnica da observação do campo e realização de grupos focais. Um primeiro grupo focal contou com a presença de nove usuários, e o segundo, com nove familiares. Foram priorizados os usuários e os familiares que tivessem participado concomitantemente das pesquisas Capsul I (2006) e Capsul II (2011). Os grupos focais são apropriados para ampliar a compreensão a respeito de um tema investigado ou a respeito de um serviço. Também podem ser utilizados para identificar mudanças que os diversos atores gostariam de ver ou as dificuldades encontradas no local investigado (GOMES; BARBOSA, 1999).

Dessa forma, os indicadores preliminares criados foram apresentados para esses grupos, para que passassem por um processo de discussão e validação. $O$ ponto central da realização desses grupos focais consistiu em os usuários e familiares discutissem os indicadores preliminares afirmando aqueles que representavam as principais mudanças ocorridas em suas vidas e apontando aqueles que seriam importantes para avaliar os resultados esperados de um serviço de saúde mental. Por fim, foram totalizados 14 indicadores qualitativos de resultado validados dentro de suas categorias. Vide a seguir.

Quadro 1. Matriz de indicadores qualitativos de resultado consensuados a partir dos grupos de usuários e familiares em Alegrete (RS), 2014

\begin{tabular}{ll}
\hline Categorias Analíticas & \multicolumn{1}{c}{ Indicadores qualitativos } \\
\hline Autonomia & - Melhor autogestão da renda \\
& - Realização das atividades do cotidiano \\
& - Maior poder de negociação \\
& - Empoderamento para enfrentamento do preconceito \\
Preconceito & - Diminuição do preconceito \\
& - Participação Social \\
Reinserção Social & - Existência de associações \\
& - Ampliação da rede social \\
& - Volta ao trabalho \\
& - Melhora na comunicação \\
& - Construção de vínculos de amizade \\
& - Melhora na qualidade das relações interpessoais \\
& - Menor necessidade de internação psiquiátrica ou não internação \\
Satisfação & - Sentimento ou sensação de bem-estar \\
\hline
\end{tabular}


Ao reconhecer a satisfação como um dos principais meios de avaliação dos resultados proporcionados pelas práticas em saúde mental, este artigo irá se ater aos indicadores qualitativos que compuseram a categoria Satisfação. As demais categorias e seus indicadores correspondentes são debatidos em posteriores publicações.

Os nomes dos participantes e a data das falas foram apresentados da seguinte maneira: Usuário 1 e ano da entrevista, por exemplo: U1 2006, ou U2 2011 ou U3 2014; Familiar 1 e ano da fala, por exemplo: F1 2006, ou F2 2011 ou F3 2014. Esta pesquisa teve financiamento próprio, e o projeto foi submetido à aprovação do Comitê de Ética em Pesquisa por intermédio da Plataforma Brasil e foi aprovado por meio do Parecer $n^{\circ}$ 753.374. Os aspectos desse estudo atendem também à Resolução CNS nº 466/2012.

\section{Resultados e discussão}

A partir da revisão do estado da arte do tema satisfação em saúde mental, muitos foram os estudos que utilizaram a satisfação como foco da avaliação dos serviços. Todavia, os estudos encontrados sobre a temática se ativeram a avaliações apenas no plano quantitativo de suas análises, o que, de certa forma, limita o processo de discussão e comparação dos resultados qualitativos encontrados neste estudo. Por outro lado, os resultados deste estudo apresentam seu ineditismo quando se pretendeu criar parâmetros qualitativos para avaliação dos resultados da atenção psicossocial proveniente da perspectiva dos próprios usuários e familiares.

Do conjunto de categorias analíticas apresentadas no quadro 1, a categoria Satisfação traz dois importantes indicadores. $\mathrm{O}$ percurso metodológico utilizado proporcionou aos usuários e familiares a possibilidade e responsabilidade de afirmar quais resultados se apresentaram como relevantes em suas vidas, indicando esses aspectos como potenciais indicadores a serem utilizados em processos avaliativos que busquem compreender a satisfação dessa população. Por isso, elegeu-se a categoria satisfação a fim de discuti-la compreendendo os seus indicadores como potenciais auxiliadores de processos avaliativos.

A categoria analítica sobre Satisfação dos usuários e familiares inseridos no serviço de atenção psicossocial (Caps) apresentada ao longo do texto é composta por dois indicadores qualitativos de resultado, 'menor necessidade de internação psiquiátrica ou não internação e sentimento ou sensação de bem-estar'.

\section{Menor necessidade de internação psiquiátrica ou não internação}

Os processos avaliativos configuram-se em importantes instrumentos com potencial de qualificação das práticas e dos serviços, dado que, de acordo com alguns estudos, a qualificação desses eixos se mostra como um elemento imprescindível à sustentabilidade da Reforma Psiquiátrica, garantindo a permanência dos usuários em seus contextos familiares e sociocomunitários, possibilitando a territorialização do cuidado e interrompendo o circuito de internações, segregações e cronificações (SILVA; DIMENSTEIN, 2014).

Pelo que foi percebido a partir dos resultados, tanto os usuários quanto os familiares se sentem satisfeitos com o serviço a partir do momento que a inserção neste culminou em uma menor necessidade de internação psiquiátrica.

O tratamento moral baseado na internação, proposto por Pinel, pouco efeito teve sobre os sujeitos que tinham transtornos mentais. Os espaços de internação psiquiátrica proliferaram a exclusão dos sujeitos em sofrimento psíquico, e esse modelo de tratamento não promoveu mudanças sociais a respeito da discriminação (PEREIRA; COSTA-ROSA, 2012).

Durante as discussões proporcionadas pela realização dos grupos focais com 
familiares dos usuários do serviço para validação dos indicadores, a questão das internações psiquiátricas se apresentou como um fator de incômodo e sobrecarga, principalmente quando havia a necessidade de os usuários serem internados em hospitais psiquiátricos fora da cidade.

Apesar de os familiares expressarem a preocupação e o sentimento de impotência no momento que as internações eram necessárias, eles também relataram de forma veemente a implicação positiva que o serviço proporciona aos seus usuários no que concerne à diminuição da necessidade de internações destes.

[...] Faz bem pra ela (o serviço), quando ela quer alguma coisa assim, quer desabafar, pra ela é bom, melhora, não fica com aquilo guardado. Graças a Deus, nunca mais ela foi hospitalizada, nem aqui nem em Passo Fundo [...]. (F1 2011).

[...] Meu filho faz uns três quatro anos que não sabe o que é baixar pra um hospital. O único problema dele é quando da gripe [...]. (F8 2014).

Portanto, a menor necessidade de internação psiquiátrica ou não internação se apresentou como o primeiro indicador qualitativo de resultado validado.

Como demonstrado na fala a seguir, o familiar percebe que os atendimentos e as atividades desenvolvidas no serviço se apresentaram como pontos auxiliares para não reinternações dos seus usuários.

[...] Claro muito bom. Não internou mais depois que ele veio pra cá. Antes, seguido ele tava no hospital e depois que ele começou a vir pra cá ele melhorou com os tratamentos, eu acho muito bom, é muito bom. Eu acho o serviço muito bom porque a maioria deles tratam tudo em casa. Antes se vivia em hospital por causa de depressão e agora não, ele tá aqui e tem toda a assistência, então ele não tem passado por essas coisas, depois que ele veio pra cá ele melhorou nisso aí, ele não vai mais [...]. (F2 2014).

As falas abaixo mostram aspectos interessantes em relação ao tratamento desenvolvido. Segundo estas, os usuários entendem que quando o tratamento é desenvolvido com qualidade, profissionais adequados e medicação de qualidade, as internações diminuem.

[...] Eu fui internado na Santa Casa de Caridade de Alegrete, que foi uma vez só. Nesse período de 5 anos não baixei mais, por motivo de eu estar com meu tratamento em dia [...]. (U10 2006).

[...] Olha, eu acho que é super bem cuidado. Porque cada oficina tem uma pessoa pra coordenar, pra cuidar. As pessoas são boas, são atenciosas, são calmas, não são estúpidas com ninguém, então eu acho que todos eles são bons, né. Todas as oficinas eu conheço todos, eu cuido também, apesar de eu ser usuário, eu tô aqui, eu tô cuidado... Eu adoeci, com 33 anos, tô com 47, com remédio novo que o Dr. me deu, então a minha vida melhorou $100 \%$, medicamento, graças a Deus curado pela saúde mental [...]. (U11 2011).

Atualmente, ainda há uma forte influência do modelo de atenção medicalocêntrico e hospitalocêntrico, em que o foco da atenção e do cuidado em saúde mental se persevera apenas na doença e nos percalços decorrentes dela. Contudo, os movimentos da Reforma da saúde mental vêm buscando interferir e transformar a lógica pertencente à prática psiquiátrica tradicional (DIMENSTEIN; LIBERATO, 2009).

As falas acima apresentaram o reconhecimento de outras formas de tratamento desenvolvidas pelo serviço, como a realização das oficinas de arteterapia e outras. A partir delas, percebe-se que o usuário reconhece outras formas para o desenvolvimento do cuidado que não somente o tratamento medicamentoso e o médico como único agente promotor do tratamento. 


\section{Sentimento ou sensação de bem-estar}

O segundo indicador qualitativo relativo à satisfação com o serviço foi o sentimento ou sensação de bem-estar. São muitos os aspectos que determinam o bem-estar, entre eles estão: a saúde, as relações interpessoais, a satisfação com o trabalho e a liberdade de expressão e política. Com a emergência do conceito de bem-estar, como é conhecido hoje, foi necessário proceder a uma distinção operacional e terminológica acerca do seu conceito (GALINHA; RIBEIRO, 2005).

Por muitos séculos, o conceito de bem-estar esteve ligado ao sentido econômico, ou seja, às contribuições dos bens e serviços que o dinheiro poderia comprar para o Bem-Estar. Somente a partir da década de 1960 que o conceito de bem-estar subjetivo passou a ser desenvolvido por Wilson (1967), atrelando-o aos conceitos de satisfação e felicidade.

De acordo com a Organização Mundial da Saúde, o conceito de bem-estar subjetivo se apresenta como uma das dimensões da saúde mental, sendo outras dimensões também a percepção de autoeficácia, a autonomia, a competência, a autoatualização do potencial intelectual e emocional, entre outros (WHO, 2001). Destaca-se também a diferenciação entre os conceitos de bem-estar subjetivo e bem-estar psicológico, em que o segundo se caracteriza por se relacionar aos conceitos de autoaceitação, autonomia, controle sobre o meio, relações positivas, propósito na vida e desenvolvimento pessoal. De acordo com Galinha e Ribeiro, o bem-estar subjetivo se apresenta como um potencial indicador de saúde mental relativo à satisfação com a vida, à felicidade e aos afetos (GALINHA; RIBEIRO, 2005).

Neste caso específico da saúde mental, o que se percebe é uma mudança do modelo assistencial, no âmbito das políticas e nas práticas dos serviços, contudo, a produção textual de base empírica ainda se faz incipiente quando se trata da avaliação dos resultados desse modelo.
De acordo com os resultados deste estudo, tanto os usuários quanto os seus familiares definiram que o sentimento e sensação de bem-estar são produzidos pelas atividades desenvolvidas pelo serviço. É sabido que estas ações de atenção psicossocial são voltadas tanto para as dimensões externas aos sujeitos quanto para a dimensão interna. As dimensões externas estariam relacionadas com a reinserção dos usuários no território, a diminuição do preconceito entre outras. As dimensões internas estariam relacionadas com as questões subjetivas e com o bem-estar emocional dos usuários e seus familiares.

Pelo que pode ser percebido, as atividades voltadas para artes, como o canto, dança e atividades festivas desenvolvidas pelo serviço, produzem sentimento e sensação de bem-estar para os usuários. Essas atividades também auxiliam na medida em que trazem uma sensação de tranquilidade e de maior potencial de escuta do outro, como relatado abaixo:

[...] A gente participa das festinhas que tem ali, tudo muito bem organizadinho. Eu tive na festa [...] já teve umas quantas festas ali. Elas dançam, elas brincam. Tudo isso vai fazendo parte da atividade da vida delas. E dançou, brincou, tava boa, bem organizada [...] tava muito bom, então tudo isso é bom para eles mesmos também. Tudo tem festa. As festinhas deles são muito boas pra eles [...]. (F4 2006).

[...] Os Caps ajudam, ajudam muita coisa, olha que quando eu vim pra cá eu não sabia nem cantar, e agora até em casa eu me pego cantado às vezes, é e aí eu digo assim não tá bom, o negócio de canta é só lá no Caps, eu chego cantando, mas às vezes eu vou me lembrar e eu aprendi lá no Caps, é beleza [...]. (U8 2011).

[...] Se desenvolve mais, até fica mais calmo e fica mais esperto até pra conversar com as pessoas, você entende melhor, aceitam e ouve melhor [...]. (F4 2014). 
Portanto, tanto os usuários quanto os familiares reconhecem as atividades festivas como atividades que promovem o bem-estar deles, a integração entre as partes e proporcionam a formação de vínculos mais fortes entre esses sujeitos.

Um estudo realizado em um Caps tipo I no oeste de Santa Catarina, em 2010, buscou identificar qual era o significado do Caps para 24 usuários desse serviço. Como resultado, obteve-se que os usuários percebem o serviço como um dispositivo que proporciona melhoras nas suas vidas a partir da me-lhora no quadro clínico, do aumento do bem-estar e um alívio para a suas angústias. Relataram também que o Caps atende às suas demandas em relação aos vínculos familiares e às relações sociais. Como conclusão, os autores descrevem que os Centros de Atenção Psicos-social representam uma melhora relevante na realidade brasileira e que a consolidação desses serviços precisa ser enfatizada. Necessita-se de uma maior cobertura desses serviços no território, visto que eles proporcionam uma redução das internações psiquiátricas e, consequentemente, a diminuição da exclusão decorrente destas (MUHL; FELTES; BITTANCOURT, 2012).

Em outro estudo realizado em Minas Gerais, o qual teve como objetivo avaliar a mudança percebida em usuários de um Caps por meio da Escala de Mudança Percebida, obteve-se como resultado que a grande maioria dos usuários (98\%) e dos familiares (96\%) respondeu que o tratamento realizado no âmbito desse serviço os ajudou a se sentirem melhor (COSTA ET AL., 2011). Dessa forma, os resultados encontrados nesta pesquisa corroboram os achados daquele estudo, evidenciando mais uma vez os resultados positivos oriundos desses serviços.

Ao pensar o bem-estar como indicador, compreende-se como um resultado positivo da reforma psiquiátrica, mas não só em relação à estabilização e reversão do quadro psíquico dos usuários, mas, sobretudo, que se possa entendê-lo no seu sentido mais amplo. Dentro da perspectiva singular de cada pessoa envolvida, destaca-se a importância de o próprio usuário avaliar o seu bem-estar e saber levar a vida da melhor maneira que lhe é possível.

\section{Considerações finais}

A partir da realização deste estudo, é confirmado que o serviço de atenção psicossocial estudado executa suas ações fundamentadas nos preceitos da Reforma Psiquiátrica e da atenção psicossocial. Este vem proporcionando uma diminuição da necessidade ou extinção das internações psiquiátricas, produzindo sentimento e sensação de bem-estar e apontando-o como um dispositivo de qualidade que reflete impactos positivos na vida tanto dos usuários quanto dos seus familiares.

Outro ponto que se destaca é o envolvimento dos familiares nesse modelo de atenção à saúde. Estes não mais se posicionam como meros expectadores do sofrimento psíquico, mas sim, passam a se envolver fisicamente e sentimentalmente, colocando-se como participantes do cuidado a esses usuários.

Esses indicadores representam uma parte simbólica, porém que se materializam na vida dos sujeitos, sendo os indicadores de resultado sobre satisfação de grande importância para o reconhecimento de uma política que preza pela liberdade, comprometimento e produção de vida, tanto para os usuários quanto para os seus familiares.

No que concerne à avaliação de serviço, tais indicadores apresentam-se como potenciais ferramentas a serem utilizadas em processos avaliativos que tenham o intuito de analisar de forma mais profunda os resultados das práticas desenvolvidas pelos 
serviços de atenção psicossocial em diferentes contextos.

\section{Colaboradores}

PFA: contribuiu substancialmente para a concepção, planejamento, análise e interpretação dos dados e elaboração do manuscrito; LPK: contribuiu substancialmente para a concepção, planejamento e participou da aprovação da versão final do manuscrito; VCCC: contribuiu substancialmente para a concepção e participou da aprovação da versão final do manuscrito; MMO: contribuiu substancialmente para a concepção e participou da aprovação da versão final do manuscrito; KLS: contribuiu substancialmente para a análise e interpretação dos dados e elaboração do manuscrito.

\section{Referências}

ALVES, P. F. Indicadores qualitativos de Atenção Psicossocial a partir da avaliação de quarta geração. 2014. Dissertação (Mestrado em Enfermagem) Pelotas (RS): Universidade Federal de Pelotas, Rio Grande do Sul, 2014.

BANDEIRA, M.; PITTA, A. M. F.; MERCIER, C. Escalas brasileiras de avaliação da satisfação (SATISBR) e da sobrecarga (IMPACTO-BR) da equipe técnica em serviços de saúde mental. J. bras. psiquiatr., Rio de Janeiro, v. 49, n. 4, p. 105-15, 2000.

BRASIL. Lei $n^{\circ}$ 10.216, de 6 de abril de 2001. Dispõe sobre a proteção e os direitos das pessoas portadoras de transtornos mentais e redireciona o modelo assistencial em saúde mental. Diário Oficial [da] União. Brasília, DF: 9 abr. 2001. Disponível em: <http://www.planalto.gov. br/ccivil_03/leis/LEIS_2001/L10216.htm>. Acesso em: 26 out. 2016.

Portaria GM/MS n 336 , de 19 de fevereiro de 2002. Define e estabelece diretrizes para o funcionamento dos Centros de Atenção Psicossocial. Diário Oficial [da] União. Brasília, DF: 19 fev. 2002. Disponível em: <http://bibliofarma.com/portariagmms-no-336-de-19-de-fevereiro-de-2002/>. Acesso em: 26 out. 2016.

COSTA, C. S. et al. A percepção de pacientes e familiares sobre os resultados do tratamento em serviços de saúde mental. Cad. Saúde Pública, Rio de Janeiro, v. 27, n. 5, p. 995-1007, maio 2011.

COSTA-ROSA, A.; LUZIO, C. A.; YASSUI, S. As

Conferências Nacionais de Saúde Mental e as premissas do Modo Psicossocial. Saúde debate, Rio de Janeiro, v. 25, n. 58, p. 12- 25, maio/ago. 2001.

DIMENSTEIN, M.; LIBERATO, M. Desinstitucionalizar é ultrapassar fronteiras sanitárias: o desafio da intersetorialidade e do trabalho em rede. Cad. Bras. Saúde Mental, Porto Alegre, v. 1, n. 1, p. 1-10. 2009.

GALINHA, I. C.; RIBEIRO, J. L. P. História e evolução 
do conceito de Bem-Estar subjectivo. Psicologia, Saúde

e Doenças, v. 6, n. 2, p. 203-214, 2005.

GOMES, M. E. S.; BARBOSA, E. F. A técnica

de grupos focais para obtenção de dados qualitativos. Educativa. 1999. Disponível em: <http://www.tecnologiadeprojetos.com.br/ banco_objetos/\%7B9FEA090E-98E9-49D2-A6386D3922787D19\%7D_Tecnica\%20de\%20Grupos\%20 Focais\%20pdf.pdf>. Acesso em: 25 jan. 2017.

GUBA, E.; LINCOLN, Y. Fourth Generation Evaluation. Newbury Park: Sage Publications, 2011.

KANTORSKI L. P. (Coord.). CAPSUL: avaliação dos CAPS da região sul do Brasil: relatório. Pelotas: Ministério da Saúde; CNPq, 2007.

CAPSUL II: segunda avaliação dos CAPS da região sul do Brasil: relatório final. Brasília, DF: Ministério da Saúde, 2011.

MINAYO, M. C. S. A utilização do método qualitativo para a avaliação de programas de Saúde, São Paulo. In: ONOCKO-CAMPOS, R. et al. (Org.). Pesquisa avaliativa em Saúde Mental: desenho participativo e efeitos da narratividade. São Paulo: Hucitec, 2008, p. 15-19.

MUHL, C.; FELTES, D. L. A.; BITTANCOURT, L. Y. B. Compreendendo um Centro de Atenção Psicossocial: a percepção dos usuários sobre um serviço substitutivo em saúde mental no Oeste de Santa Catarina, Brasil. Unoesc e Ciência, Joaçaba, v. 3, n. 1, p. 61- 68, jan./jun. 2012.
PEREIRA, E. C.; COSTA-ROSA, A. Problematizando a Reforma Psiquiátrica na atualidade: a saúde mental como campo da práxis. Saude soc., São Paulo, v. 2, n. 4, p. 1035-1043, dez. 2012.

PERLMAN, C. M. et al. Development of mental health quality indicators (MHQIs) for inpatient psychiatry based on the interRAI mental health assessment. BMC Health Services Research, London, v. 13, n. 15, p. 1-12, jan. 2013.

SILVA, M. L. B.; DIMENSTEIN, M. D. B. Manejo da crise: encaminhamento e internação psiquiátrica em questão. Arquivos Brasileiros de Psicologia, Rio de Janeiro, v. 66, n. 3, p. 31- 46, 2014.

WILSON, W. Correlates of avowed happiness.

Psychological Bulletin, Washington, DC, v. 67, p. 294-

306, abr. 1967.

\section{WORLD HEALTH ORGANIZATION (WHO) The}

World Health Report 2001: Mental health - New understanding, new hope. Geneva: WHO, 2001. Disponível em: <http://www.who.int/whr/2001/en/>. Acesso em: 26 out. 2016.

\footnotetext{
Recebido para publicação em abril de 2016

Versão final em setembro de 2016

Conflito de interesses: inexistente

Suporte financeiro: não houve
} 